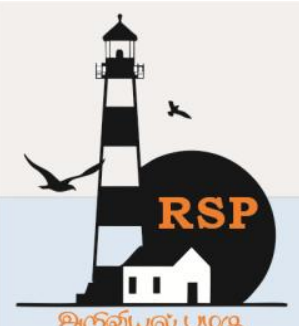

INTERNATIONAL RESEARCH JOURNAL ON ADVANCED SCIENCE HUB

RSP SCIENCE HUB

(The Hub of Research Ideas)

Available online at www.rspsciencehub.com

Special Issue of First International Conference on Management, Science and Technology (ICMST 2021)

\title{
Probabilistic Model to Predict the Fire Risk Incidental Duration
}

Nivedika ${ }^{l}$, Prateek Papriwal ${ }^{2}, P . V$. Ramana $^{3}$

${ }^{1,2,3}$ Dept. of Civil Engineering, Malviya National Institute of Technology, Jaipur, India.

pvramana.ce@mnit.ac.in ${ }^{3}$

\begin{abstract}
Based on the statistical approaches used in the research, it can be said that Jaipur is most at risk from multiple fire incidents during peak hours, which occur on weekends in the summer season from 9 am to $10 \mathrm{pm}$. As a result, the danger of several fires is 44.5 percent higher during peak hours than off-peak hours. 1.236 is Cox's risk ratio for the weekend, which concluded that the risk for event events on weekends is $23.6 \%$ higher than the days of the week. For the summer season, the Cox hazard rate is calculated to be 1.210. The result explains that winter is less likely to have multiple fires and that the summer season is $21 \%$ more likely to have multiple fires or a higher fire hazard than the winter season. Currently, the fire hazard classification method used in the northern part of the south India, where forest fires are the worst in Asia as in India, only uses climate variables without considering fire markings. According to the meteorological classification of forest fire risk, the difference between the occurrence of fire and the risk of fire, the method issued by the National Meteorological Administration to predict the possibility of forest fire through the link between the occurrence of forest fires and climate change is more obvious in the north than in the south. In recent years, due to the strict prohibition on fire signs, the number of fire signs in the area has been greatly reduced. Therefore, there is a big difference between the estimated fire hazard methods and the actual fires. To solve this problem, a probabilistic method was developed that predicts the likelihood of fire by introducing ignition components (IC) into the National Fire Classification System of the India to indicate the consequences of fires and meteorological fuel composites in the fire. The ability and accuracy of the new method in the area were evaluated. The results show that the method is suitable for this area. The IC or the modified IC can be applied to show the impact of the air-conditioning fuel complex on the fire and divide the fire risk into a smaller number of periods. The IC can determine the fire hazard level and related alarm level in the activation area. The same principle can be used to reduce similar differences between the actual occurrence of fires and fire risks in other countries.
\end{abstract}

Keywords: Fire incident, Weekdays, Hazard Models, Peak and Peak-off hours.

\section{Introduction}

The fire incident will be four minutes long, excluding one minute for emergency decisionmaking time and one minute and twenty seconds for fire fighters' preparation time (turn out time), reducing the overall interval to six minutes and twenty seconds. It strongly encourages fire departments to analyze and examine the amount and type of fire events occurring at various sites throughout a fire district (e.g., high-rise buildings, manufacturing enterprises, schools, hospitals, etc.) and to ensure that proper fire risk assessments and the right number of firefighters are provided to fire departments. One can recognize that once the likelihood of a fire incident is high or low, seek out the chance and survival analysis. Early fire 
research focused on blazing service intervals and primarily on fire events. The well-known "inverse root laws" of Kolesar and Blum, for example, illustrate the mathematical relationship between the typical response distance and the range of fireside stations to perceive the link between fire engine travel distance and reaction time. Each study looked at fires in New York City and tried to figure out what the appropriate number and location of fire stations would be. Knowing the definition of fireside danger is helpful when evaluating fire risk assessment.[1-5]. A fire hazard has two parts that are weighed against each other: one is the risk of a fire occurring, and the other is the size of the fire's repercussions. Because of the cutting and attaching equipment, a metal fabrication workshop, for example, has a high risk of fire. If the housework is done properly and there are no flammable materials available, a fire is unlikely to spread, and the risk of serious consequences is low. The chance may be thoughtabout to be traditional or perhaps low. In an exceeding polysaccharides exceedingly booth, a happening is extremely probably thanks to the merchandise used and also the instrumentation needed for the process. The results are very high as a result of any fireplace would have a speedy development; consequently, it might have to be compelled to be thought-about a high risk. These may be reduced to acceptable levels by varied methods, with intelligent ping, specially designed electrical apparatus, equipment set far from the chance, and compressed gasses employed in the processes, piped to the risk from a bulk storage or centrally located position. Mark J. Hancock, Christopher G. Maher (2014)[1], the researchers gave an idea and introduction concerning the survival analysis during this study. The same that Survival analysis could be an applied math approach want to study time to active interest. The approach extremely applicable to the manual medical aid discipline, nonetheless, is not used. The results gift an easy, straightforward survival analysis geared toward clinicians and researchers within the manual therapy field. Specifically, researchers aim to 1) describe things wherever survival analysis is acceptable to use 2) describe distinctive characteristics of survival knowledge together with the censoring of participants 3 ) describe analysis strategies for survival data including log-rank take a look at and Cox regression 4) describe the interpretation of results from survival analyses together with survival plots and hazard ratios. Enis Cetin and blue blood Merci (2016)[2] Study urged that to avoid large-scale fire, timely and correct fire detection is essential, and smoke damage, timely and correct fire is detected, the higher the possibilities are for survival. [6-10]. However, early detection is crucial; however, it is additional to fully understand the fire development and the location. Nevertheless, most video fire detection (V.F.D.) approaches ring the bell and cannot model fire evolution note that data concerning the hearth circumstances is not available). The fire not during this chapter focuses on each problem. It presents man problem modal/multi-sensor analysis techniques that have verified helpful quick and correct anals of valuable fire characteristics (flame and smoke spreading) with video and alternative forms of volume sensors. These characteristics, in turn, may be used for fire-spread forecasting. Godfred Yeboah and Peter Y. Park (2018) [3] during this study discovered that the pre-emptive allocation of fireside engines for planned short-run periods from one rigorously selected fire district to a different might facilitate fire districts to retort to multiple incidents a lot of expeditiously and effectively by increasing the utilization of a municipality' existing resources. This study used survival analysis (the Kaplan-Meier figurer and Cox hazard model) to work out the time once the chance of multiple emergencies was expected to be highest in an exceedingly "demand" fire district and lowest in a "supplier" district. Traditional machine learning methods based on data-driven models to predict the area burned by forest fires have a common feature in that they incorporate regression analysis, correlation,and/or statistical distribution into their calculation processes, which usually have hidden layers and Rock that is difficult to penetrate.[11-15]. The calculations of these models are usually not transparent enough to retrieve data quickly and in detail from their modelling datasets. This research specifically designed and developed a newly developed optimized data comparison model with $4^{\text {th }}$ International Conference on Reliability, Safety and Hazard (ICREASH-2019) IIT MADRAS transparently opened window for prediction. This 
method collects data from the well-studied forest fire data set, where it is first used to estimate forest fires. It avoids the data set by using correlations, regression or statistical relationships between variables. The statistical data can easily display each forecast's detailed information. The historic fire obviously coincides with every event. This makes the model more suitable for granular data analysis applications needed to understand historical wildfire data sets better.[16-20].

\section{Methodology:}

In this paper, many fireplace incidents information is taken. The hazard analysis model for risk prediction is ready victimization cox hazard tools to mitigate future ruinous and evolution fire incidents. Cox hazard analysis is that the new technique in Disaster Assessment \& Mitigation. The hazard performance is a vital characteristic of a period distribution. It indicates that the chance of failure varies with age or time of interest in most applications. Previous info regarding the form of the hazard function will facilitate guide model selection. Finally, if factors poignant in an individual's lifetime vary over time, approaching modeling through the hazard performance is essential. The Cox hazards model depicts the applied mathematics link between numerous input variables (such as the season of the year and the type of incident) and the time it takes for numerous incidents to survive. We usually build our hazards model to represent the possibility of multiple incidents in a very specific fire district. [2125].The hazard function at a given time $t$ denoted by $h(t)$ was obtained as follows:

$$
\begin{aligned}
& h_{j}(t)= \\
& h_{0}(t) \exp \sum_{i} \sum_{j \leq t} \exp \left(\beta_{i} X_{i j}\right)
\end{aligned}
$$

Where $h_{j}(t)$ is the hazard at time $t_{j} ; h_{0}(t)$ is the baseline hazard; $X_{i j}$ represents the input variable $\mathrm{i}$ at a period of $t_{j}$, and $\beta_{i}$ is the risk of the corresponding input variable i a fire district. If $\beta_{i}=0$, the ith input variable is not statistically significant. However, if $\beta_{i} \neq 0$, the ith input variable has a statistically significant relationship with the occurrence of multiple incidents. Taking natural logarithms to both sides of the above equation:

$$
\begin{aligned}
& \ln \left\{\frac{h_{j}(t)}{h_{0}(t)}\right\} \\
& =\sum_{i} \sum_{j \leq t} \llbracket \exp \left(\beta_{i} \rrbracket X_{i j}\right)
\end{aligned}
$$

If $[\beta \Omega$ is positive, the likelihood of an incident occurring as a result of the associated input variable is high. A negative value of $[\beta]$ represents a coffee risk of the event occurring for that particular input variable. The hazard magnitude relationship is that the exponential of

$\square \beta \rrbracket$ associate degreed is defined as the relative hazard loves a unit change within the associated input variables. As a result, if an input variable's hazard ratio is less than one, the input variable has very little influence on the risk of an event occurring. Even so, the risk becomes significant once the hazard magnitude relationship exceeds 1 . To test this type of question, three standard checks will be used: the chance ratio (L.R.) test, the Wald test, and the Lagrange number test (sometimes referred to as a score test). Because one model might be aforementioned to be nested at intervals the other, these tests are often stated as tests for changes among nested models. The null hypothesis is that the smaller model is the "true" model in all three tests; an outsized test statistic shows that the null hypothesis is wrong. Despite the fact that all three tests cover the same basic question, they are slightly different. As a result, this section will explain how to run these checks as well as their similarities and variances. These tests are broad and can be used to evaluate a variety of hypotheses, such as whether changing a parameter has a significant impact on model fit.

\section{Problem Statement:}

A fireplace will begin rapidly and still rage till its fuel supply is depleted, destroying homes and property, inflicting injuries, and taking lives at intervals in a matter of seconds. In some cases, the ensuing losses are extraordinary, causing many deaths, widespread harm to property and contents, and vital environmental impacts. Most cities face many challenges once responding to multiple coinciding fire emergency calls, and Jaipur is one in each of them. The matter seems to arise additional usually than may well be expected. The chance period for preset short-run periods of incidents can be calculated. Rajasthan is that the 


\section{www.rspsciencehub.com}

largest state of the Republic of India, experiencing perennial forest fires. Jaipur Terminal fire in 2009 was a severe fire disaster. The fireplace raged for eleven days, killed 11 people, and lost value to Rs 2.80 billion.

\section{Results and discussion:}

\subsection{Hearth risk analysis:}

Applied mathematics model applied mathematics approaches to see the danger assessment for the various time incidents and multiple calls incidents within the Jaipur city area. With the Cox hazard model, a hazard model is developed for the town to seek out the bottom of the very best risk amount for the fire. Numerous statistical tests that are done are illustrated in the following sections.

\subsection{Kaplan Meier calculator}

The survival time curves generated by the metric linear unit estimator depict the possibility of several occurrences occurring in the city, which is also known as the Survival probability analysis. The survival possibilities decrease over time due to the productive analysis, and it is a non-parametric test. Metric linear unit calculator finds out the foremost probable risk incident classes within the Jaipur space: the analysis area of this study. When having results for all three categories, the Cox hazard model for hearth risk assessment is placed in the Cox hazard model. The upper or highest likelihood of several events is shown by the lower or lowest curve in each of the three comparisons. The bottom curve offers the worth of additional risk throughout that specific category, indicating higher risk. The Kaplan-Meier calculator curves for the class are shown in Figures 1,2, and 3: (a) Peak/off-season hours; (b) Weekdays; (c) Season of the Year

\subsubsection{Peak/ off-season hours}

Figure 4.1 shows the graphs between Peak hours and off-season hours. The lower or lowest curve, as compared, shows the upper or highest likelihood of multiple incidents. The lowest curve offers the worth of additional risk throughout that specific category, indicating higher risk. Here lowest curve represents the off-peak hours; thus, It should be noted that a comparison of the curves reveals that numerous fire event occurrences inside the town are more likely during peak hours than non-peak hours. The above result shows that the danger is more significant or higher in Peak hours
Volume 03 Issue 06S June 2021

than the off-season hours; it will represent a number perform of primary hazard functions in cox analysis. Time is taken as one hour; that is why the graphs are planned between survival analysis likelihood versus 1 hour (60 minutes) study time.

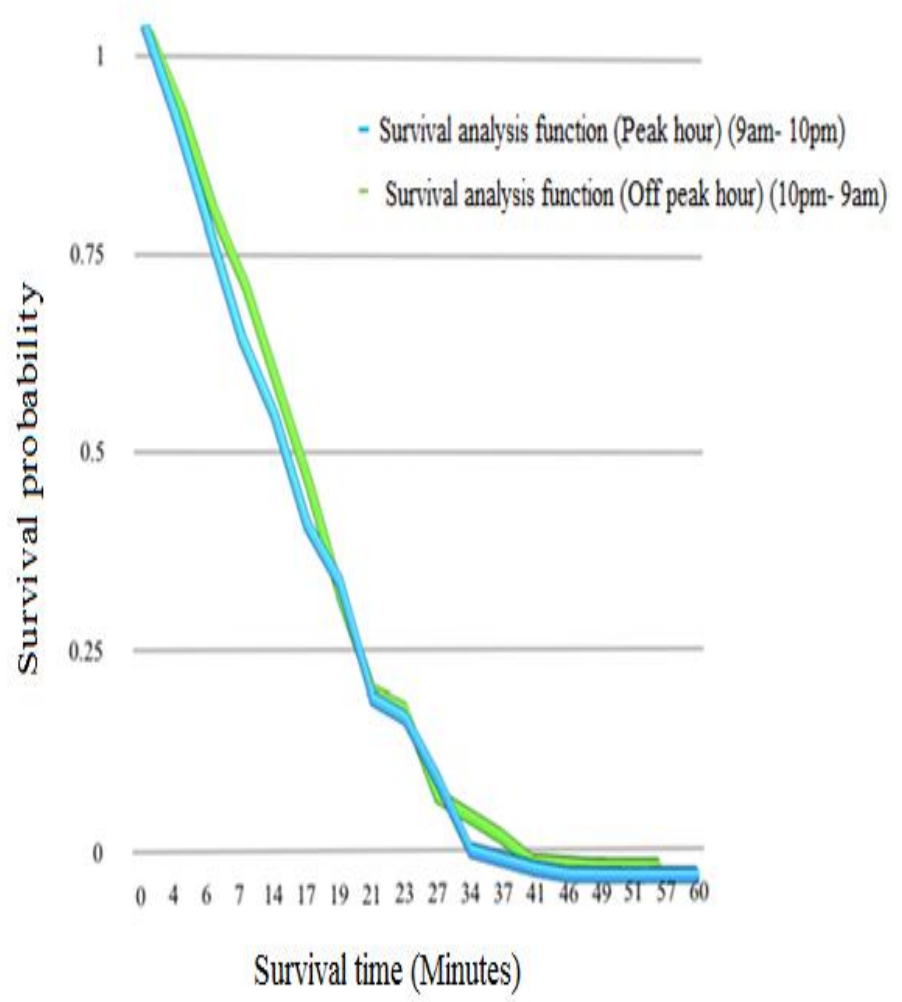

Fig.1. Kaplan Meier estimator for survival probability for peak/off-peak hours

\subsubsection{Days of the week}

Figure 4.2 shows the graphs between days of the week, i.e., Weekdays and weekends, as a result of the upper or highest curve as compared shows the lower or lowest likelihood of multiple incidents. The Lowest curve offers the worth of many risks throughout that particular time of the class, indicating higher risk. Here, the lowest curve represents the weekends; therefore, The comparison of the curves reveals that numerous hearth event occurrences within the town are more likely to occur on weekdays than on weekends. As a result of the danger is more significant or higher at the weekend than on the weekdays. It will represent a multiplier factor performing the essential hazard function in cox analysis. One hour is taken because of the study time; that is why the graphs are premeditated between survival analysis likelihood versus 1 hour (60 minutes) study time. 


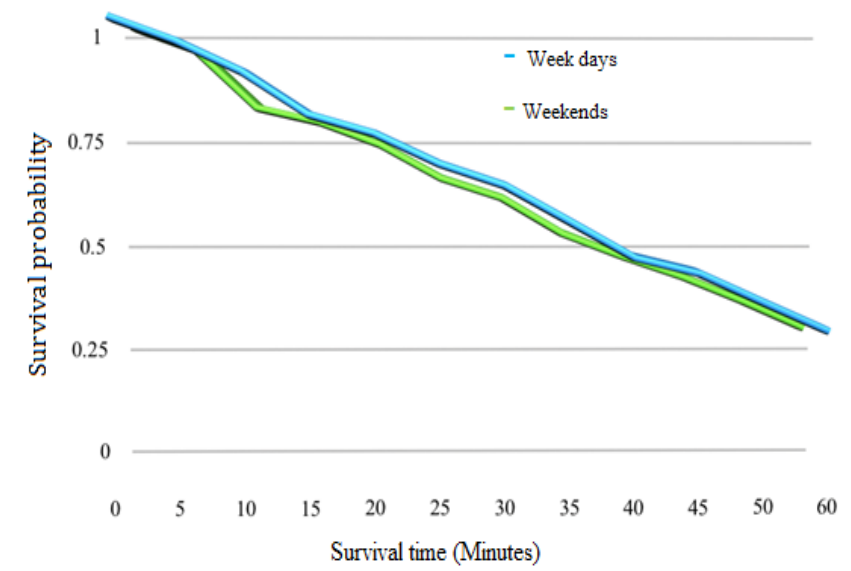

Fig.2. Kaplan Meier estimator for survival probability for weekends/weekdays

\subsubsection{Season of the year}

Figure 4.3 shows the graphs between the season of the year, i.e., summer and winter. Solely two seasons are taken here to stay in mind the Jaipur study area. As a result of the lower or lowest curve compared shows the upper or highest chance of multiple incidents. Therefore the lowest curve offers many risks throughout that specific time of the class, indicating higher risk. Because the lowest curve indicates the summer season, it is frequently stated that a comparison of the curves reveals that several fireplace event occurrences within the town are more likely during the summer season than during the winter season. As a result of the danger is more significant or higher at the weekend than on the weekdays. It will represent a multiplier factor operating of the fundamental hazard function in cox analysis. Time is taken as one hour; that is why the graphs are aforethought between survival analysis chances versus 1 hour (60 minutes) study time. 4.3 Equality takes a look at survival analysis Log-Rank, and Wilcoxon tests are for equality test for survival. These tests determine whether the differences in the KaplanMeier estimator's results for various periods and different sorts of occurrences or classes were significant or not. Confidence limits are taken as $95 \%$. The degree of freedom for all 3 categories is $(\mathrm{k}-1)=2-1=1$, wherever $\mathrm{k}$ represents the worth of forms of incidents taken within the one category, i.e., for a season of the year, the primary is summer, second is winter. Therefore here, D.O.F. is going to be adequate to 1 . Table.1 shows the value of calculated values for Chi-square, D.O.F., and the corresponding p-value. For the numerous results of the kilometer estimator, the p-value ought to be under $5 \%$. It is often aforesaid that the height hours, weekends, and summer season are the upper risk or multiple incidents of fireside prevalence classes. Here it can be noticed that the $\mathrm{p}$-value for each the takes a look at statistics are under $5 \%$, or it can be said that the p-value is a smaller amount than 0.05 . It may be concluded that significant variation exist between the KaplanMeier estimators for all three categories of each input variable considered.

Table.1: Kaplan Meier estimator equality test results

\begin{tabular}{|c|c|c|c|}
\hline Test & ChiSquare & DOF & p-Value \\
\hline Log-Rank & 8.736 & 1 & 0.0021 \\
\hline Wilcoxon & 13.987 & 1 & 0.001 \\
\hline
\end{tabular}

\subsection{Cox Hazard Model}

All three were incorporated to the Cox hazard model after it was discovered that Kaplan Meier's estimate produced significant findings for all three categories and that the likelihood of many fire occurrences in Jaipur city is higher during the summer, weekends, and peak hours. The intent of the Cox hazard model research was to look at the impact of each of the three variables on the likelihood of multiple events in Jaipur. Hazard model predicted for the fire incidents based on the different classification is:

$$
\begin{aligned}
& \ln \left\{\left(h_{-} j(t)\right) /\left(h_{-} 0(t)\right)\right\} \\
& =\exp \left((a) \times X_{-}(p e a k \text { hour })+b \times X_{-}\right. \text {weekend } \\
& +c \\
& \left.\times X_{-} \text {summer }\right) "
\end{aligned}
$$

Where $h_{j}(t)=$ The hazard at a specific time $t_{j}$ $h_{0}(t)=$ The baseline hazard

$X_{\text {peak hour }}, X_{\text {weekend }} \& X_{\text {summer }}$ represent the input variable over $t_{j}$ time period, and $\mathrm{a}, \mathrm{b}$ and $\mathrm{c}$ are the unknown parameters.

These parameters were found out with the maximum likelihood ratio method. The standard 
www.rspsciencehub.com

error and chi-square values for the parameters are calculated, and the hazard ratio and corresponding p-value for the parameters.

\section{Table.2: Maximum likelihood test results for} Cox hazard model

\begin{tabular}{|c|c|c|c|c|c|}
\hline Parameters & $\begin{array}{c}\text { Parameters } \\
\text { estimated }\end{array}$ & Std Error & Chi Square & p-Value & $\begin{array}{c}\text { Hazard } \\
\text { ratio }\end{array}$ \\
\hline Peak Hours & 0.436 & 0.067 & 16.687 & 0.0012 & 1.445 \\
\hline Weekend & 0.165 & 0.083 & 5.012 & 0.043 & 1.236 \\
\hline Summer & 0.204 & 0.069 & 8.992 & 0.0021 & 1.210 \\
\hline
\end{tabular}

Table. 2 offers the worth of parameters a, b \& c calculated for the variables $X_{-}$(peak hour), X_weekend \& X_summer. For all 3 categories, one will see that the value of standard error is extremely less. The $\mathrm{p}$-value for all the parameters is a smaller amount than $5 \%$ or 0.05 . It is often all over from here that the null hypothesis can be rejected, that is $\left[\beta \Sigma_{-} \mathrm{i}=0\right.$, or the shorter model is the best one. Thus it can be the same that \& $\beta \Omega_{\_} i \neq 0$ or additional variable tends to relinquish the simplest match model for the hazard analysis. Furthermore, these three variables contribute to an increase in the likelihood of multiple hearth accidents in the space. that the Cox hazard model for the analysis area is often written as:

$$
\begin{aligned}
\ln \left\{\left(h_{-} j(t)\right) /\right. & \left.\left(h_{-} 0(t)\right)\right\} \\
& =0.436 \times \varangle X \nabla_{-}(\text {peak hour }) \\
& +0.165 \times \varangle X \nabla_{-} \text {weekend } \\
& +0.204 \\
& \times \nabla X \nabla \_ \text {summer " }
\end{aligned}
$$

Where $h_{j}(t)=$ The hazard at a given period $t_{j}$

$h_{0}(t)=$ The baseline hazard

The hazard result shows that at a given amount of time which parameter affects more. The baseline hazard, the quantitative hazard relation for all 3 variables, is additionally calculated. For peak hours, the cox hazard ratio is noticed at 1.445 . The
Volume 03 Issue 06S June 2021

result implies that the danger of occurring multiple fire incidents in Peak hours is $44.5 \%$ over the nonpeak hours. 1.236 is that the Cox hazard quantitative relation for the weekend is that the events' risk of events at weekends is $23.6 \%$ more than the weekdays. For the summer season, the cox hazard ratio is found out 1.210. thus it is often the same that the winter is a smaller amount probably to own multiple fire risk incidents and summer is $21 \%$ additional likely to have multiple fire incidents or higher risk of fireside events.

\subsection{Fit model tests}

Likelihood, Wald, and Score tests are done as the goodness of fit tests. 95\% confidence interval is taken in the study. To check the null hypothesis, these tests are done so that the significance of the cox hazard model is found out. Table. 3 shows the result of the analysis.

\section{Table.2: Fit model test result for hazard model}

\begin{tabular}{|c|c|c|c|}
\hline Test & Chis Square & DOF & p-Value \\
\hline Likelithood Ratio & 71.476 & 3 & 0.0011 \\
\hline Score & 69.023 & 3 & 0.00178 \\
\hline Wald & 68.923 & 3 & 0.00113 \\
\hline
\end{tabular}

Every goodness of match test rejects the null hypothesis (that all $\beta \mathrm{i}=0$ ) at the 95 percent confidence level, as the p-value for each of the three check statistics is less than 0.05 . The result suggests that a minimum of one $\beta i \neq 0$ and the parameters calculable for peak hours ( 9 am-10 pm) for summer and weekend incidents are applied monthly vital at the $95 \%$ confidence level.

\section{Conclusions}

According to the statistical methodologies used in the study, Jaipur has a higher chance of several fire occurrences during peak hours, which are nine a.m. to ten p.m. on weekends during the summer season. Also, for peak hours, the cox hazard quantitative relation is realized to be 1.445 . The result suggests that the chance of occurring multiple fireplace incidents in Peak hours is $44.5 \%$, quite the off-season hours. 1.236 is that the 


\section{www.rspsciencehub.com}

cox hazard ratio for the weekend terminated that the events' risk of events at weekends is $23.6 \%$ on top of the weekdays. For the summer season, the cox hazard ratio is calculated as 1.210 . The result explains that the winter is a smaller amount, seemingly to own multiple fire risk incidents. The summer season is $21 \%$ additional, seemingly to own multiple fire incidents or a higher risk of fireplace events than the winter season.

\section{References}

\section{Journals}

[1].Mark J. Hancock, Christopher G. Maher, Luciola da Cunha Menezes Costa, Christopher M. Williams (2014), A guide to survival analysis for manual therapy clinicians and researchers, Manual therapy vol. 19, 511-516

[2].Enis cetin, B. Merci, Osman Gunay, Behcet Ugur Toryin, Steven Verstockt (2016), Methods and Techniques for fire detection, Academic press publications

[3].Godfred Yeboah, Peter Y. Park (2018), Using survival analysis to improve pre-emptive fire engine allocation for emergency use, Fire safety journal 97, 76-84

[4].N.C. Simpson, P.G. Hancock, Fifty years of operational research and emergency response, J. Oper. Res. Soc. 60 (2009) S126-S139.

[5].S. Yang, M. Hamedi, A. Haghani, Online dispatching and routing model for emergency vehicles with area coverage constraints, Transport. Res. Rec., J. Transport. Res. Board (1923) (2005) 1-8.

[6].E. Aktas, O. Ozaydin, B. Bozkaya, F.U. Sule Onsel, Optimizing fire station locations for the Istanbul metropolitan municipality, Interfaces 43 (3) (2013) 240-255.

[7].A.T. Murray, Optimizing the spatial location of urban fire stations, Fire Saf. J. 62 (2013) 6471.

[8].M. Kazemia, M.M. Kunt, I. Aghayanc, R.J. Larijani, an Optimization model for fire station location based on G.I.S. and Python: a case study in North Cyprus, Appl. Mech. Mater. 330 (2013) 1059-1064.

[9].J. P Erez, S. Maldonado, H. Lo pez-Ospina, A fleet management model for the Santiago fire department, Fire Saf. J. 82 (2016) 1-11.

[10]. R. Alanis, A. Ingolfsson, B. Kolfal, A Markov chain model for an E.M.S. system
Volume 03 Issue 06S June 2021

with repositioning, Prod. Oper. Manag. 22 (1) (2013) 216-231.

[11]. W.S.S. Lam, Z.C. Zhang, H.C. Oh, Y.Y. $\mathrm{Ng}$, W. Wah, M.E. Hock Ong, Reducing ambulance response times using discrete event simulation, Prehosp. Emerg. Care 18 (2) (2014) 207-216.

[12]. W.S.S. Lam, J. Zhang, Z.C. Zhong, H.C. Oh, J. Overton, Y.Y. Ng, W. Wah, M.E. Hock Ong, Dynamic ambulance reallocation for the reduction of ambulance response times using system status management, AJEM (Am. J. Emerg. Med.) 33 (2015) 159-166.

[13]. K. Schneeberger, F.F. Doerner, A. Kurz, A. Schilde, Ambulance location and relocation models in a crisis, Cent. Eur. J. Oper. Res. 24 (1) (2016) 1-27.

[14]. J.L.Vile, J.W.Gillard, P.R.Harper, V.A.Knight, Time-dependent stochastic methods for managing and scheduling emergency medical services. Res. Health Care 8 (2016) 42-52.

[15]. A.J. Smith, P. Dieppe, K. Vernon, M. Porter, A.W. Blom, Failure Rates of Stemmed Metal-on-metal Hip Replacements: Analysis of Data from the National Joint Registry of England and Wales, vol. 379, 2012, pp. 1199.

[16]. C.S. Williams, N.C. Durham, Survival analysis: an applied introduction, in Proceedings of the South East S.A.S. Users Group, SESUG, St Pete Beach, FL, U.S.A., 2008, pp. 1-3 (23 de junio de 2013), http://analytics.ncsu.edu/sesug/2008/ST147.pdf.

[17]. Aishwarya Narang, $4^{\text {th }} \quad$ International Conference on Reliability, Safety and Hazard (ICREASH-2019) IIT Madras

[18]. M.A. Cleves, W.W. Gould, R.G. Gutierrez, An Introduction to Survival Analysis Using Stata, Revised Edition, Stata Press, College Station, Texas, 2004, pp. 1-10.

[19]. J.P. Klein, M.L. Moeschberger, Survival Analysis Techniques for Censored and Truncated Data, second ed., Springer-Verlag New York, Inc., New York, NY, 2003, pp. 2150 . 
[20]. B. Efron, Logistic regression, survival analysis, and the Kaplan-Meier curve, J. Am. Stat. Assoc. 83 (402) (1988) 414-420.

[21]. Aishwarya Narang, International Conference on Advances in construction materials and structures (ACMS-2018) in IIT Roorkee

[22]. S.V. Masten, R.D. Foss, Long-term effect of the North Carolina graduated driver licensing system on licensed driver crash incidence: a 5year survival analysis, Accid. Anal. Prev. 42 (2010) 1648-1651.

[23]. Ayush Meena, P.V. Ramana (2021), Assessment of structural wall stiffness impact due to blast load Materials Today: Proceedings, Elsevier Publication.

[24]. Anamika Agnihotri, Ajay Singh Jethoo, P.V. Ramana, Mechanical properties of unprotected recycled concrete to fiery-hot, Materials Today: Proceedings (2021), ISSN 2214-7853.

[25]. Surendranath, Arigela, and P. V. Ramana. (2021). "Recycled materials execution through digital image processing." Materials Today: Proceedings. 